\title{
DE L'UTILISATION DES LOIS DE LA MÉCANIQUE GÉNÉRALE A L'ÉTUDE DES DIATOMÉES (BACILLARIOPHYCEAE) : JUSTIFICATION.
}

\section{J. BERTRAND}

42 rue de Malvoisine, 45800 Saint-Jean-de-Braye, France.

\section{RÉSUMÉ}

Bien que les équations de la mécanique générale aient été utilisées depuis un certain temps dans l'étude des micro-organismes, il ne semble pas que l'on ait douté de leur validité. L'auteur, dans ce premier article, s'interroge et démontre que la pression hydrostatique a une influence négligeable sur le comportement des diatomées raphidées benthiques mobiles. II démontre également que les équations simplifiées de la mécanique statique décrivent correctement le comportement des diatomées raphidées dans leur milieu aquatique.

Mots-clés : bacillariophycées, centre de gravité, diatomées, hydrostatisme, mécanique, mouvement, statique.

\section{ABOUT THE USE OF GENERAL MECHANIC LAWS FOR THE STUDY OF DIATOMS (BACILLARIOPHYCEAE) : JUSTIFICATION.}

\begin{abstract}
The equations of general mechanics have been used for some time, for the study of microorganisms, their validity has always been deemed as certain. In this first article, the author raises the questions and shows that the hydrostatic pressure has an insignificant influence on the behaviour of the moving benthic raphid diatoms. He also demonstrates that the simplified equations of static mechanics describe properly the behaviour of the raphid diatoms in their aquatic environment.
\end{abstract}

Key-words : bacillariophycees, centre of gravity, diatoms, hydrostatism, mechanics, movement, static.

\section{INTRODUCTION}

L'utilisation des équations de la mécanique générale dans l'étude des diatomées n'est pas récente, puisque HARPER et HARPER (1967) les ont employées dans le but de définir les forces d'adhérence de ces algues sur le substrat. Pour la mesure de ces forces, les auteurs ont utilisé les équations de la résistance des matériaux, appliquées à des fibres de verre de 20 microns dont ils mesuraient la flexion. Ils en déduisaient les efforts correspondants. Puis, EDGAR (1982), au plan théorique, utilisa les équations de la mécanique des fluides pour étudier le comportement des diatomées en fonction du milieu.

L'étude du comportement des diatomées planctoniques dans les eaux turbulentes a été entreprise par SMAYDA et BOLEYN (1965), SMAYDA (1970), WALSBY et REYNOLDS (1980), REYNOLDS (1984), en employant les lois de la mécanique des fluides. Plus récemment, nous 
avons utilisé les équations de la mécanique générale pour l'étude du mouvement des diatomées (BERTRAND, 1991, 1993b, 1995), et les équations de la mécanique des fluides (BERTRAND, 1993a) pour la détermination de la densité des diatomées. Par ailleurs, dans le domaine des recherches fondamentales en biologie, nous devons mentionner les études de LENGELER (1990) sur la nage des bactéries. Également, ISHIJIMA et al. (1991), KUO et SHEETZ (1993), FINER et al. (1994) mesurent les efforts développés et les déplacements lors du basculement d'une seule tête d'actine en présence de myosine, qui sont à l'origine de la contraction musculaire. Toutefois, sans aller aussi loin dans l'infiniment petit, on peut se demander si l'application des lois de la mécanique générale statique et dynamique est judicieuse pour étudier les mouvements des diatomées. Nous ne pensons pas que ces réflexions aient reçu une réponse, du moins à notre connaissance. Peut-on impunément les appliquer à des micro-organismes ?

Nous tentons, dans ce premier travail, de justifier l'emploi de la mécanique générale dans l'étude du mouvement des diatomées. La comparaison des phénomènes observés avec un calcul théorique basé sur les lois de la mécanique générale, nous permettra de déterminer si celles-ci décrivent correctement ces phénomènes.

Un rappel succinct des lois de l'hydrostatisme nous semble cependant nécessaire. Dans un liquide au repos, nous sommes en présence de 3 familles de force : les forces moléculaires, la gravitation, la force hydrostatique.

\section{Les forces moléculaires se subdivisent en 3 classes :}

a) Le mouvement brownien : agitation des molécules d'une manière anarchique, non orientée. Leurs efforts s'annulent et le corps reste en équilibre sous les chocs.

b) La viscosité : c'est la force moléculaire de résistance aux mouvements. Elle est propre à chaque type de liquide et constante dans certaines limites de température et de pression.

c) La tension superficielle : n'agit que sur les couches ultimes des molécules de surface d'un liquide. Elle est inopérante à l'intérieur du liquide.

\section{La force de gravitation}

Elle agit sur tous les corps ayant une masse. Elle agit verticalement, toujours dirigée vers le centre de la terre. Elle imprime une accélération constante lors de la chute d'un corps dans le vide, et une vitesse constante lors de la chute d'un corps dans un milieu visqueux, gaz ou liquide, suivant la loi de Stokes. La vitesse dépend de la densité relative du corps par rapport au milieu, et de ses dimensions. Elle a pour point d'application le centre de gravité du corps.

\section{La force hydrostatique (principe d'Archimède)}

Elle agit verticalement à l'opposé de la gravitation, sur tous les corps immergés. Elle est fonction du volume du corps immergé et a pour point d'application le centre de poussée, qui est le centre de gravité du volume immergé. Pour un corps homogène immergé dans un liquide homogène, le centre de gravité $\mathrm{Cg}$ et le centre de poussée $\mathrm{Cph}$ sont confondus (Fig. 1a). Le corps est en équilibre indifférent, dans toutes les orientations spatiales. Si la force de gravitation Fg est supérieure à la force de poussée Fph, le corps s'enfonce dans l'eau. La force résultante est la différence : $\mathrm{Fr}=\mathrm{Fg}$ - $\mathrm{Fph}$. Pour un corps hétérogène immergé dans un liquide homogène, la position du centre de gravité est différente de celle du centre de poussée (Fig. 1b). Le corps est en déséquilibre. II se crée un couple de rotation : $\mathrm{Cr}$, égal à ( $\mathrm{Oa} \times \mathrm{Fph}+\mathrm{Ob} \times \mathrm{Fg}) \mathrm{Cos} \alpha$, qui tend à faire pivoter le corps autour du point d'équilibre $O$ jusqu'à l'alignement vertical des centres de gravité et de poussée.

C'est donc dans les pivotements polaires verticaux des diatomées, si celles-ci sont hétérogènes, que l'action de la pression hydrostatique devrait être observée, en accélérant ou en freinant l'action propre de la diatomée mono ou bi-raphidée douée de mouvement. Nous pensons que les lois de la mécanique générale doivent pouvoir s'appliquer car, avec les diatomées, nous avons des corps rigides indéformables constitués par une boîte creuse de silice : le squelette, appelé « frustule». 

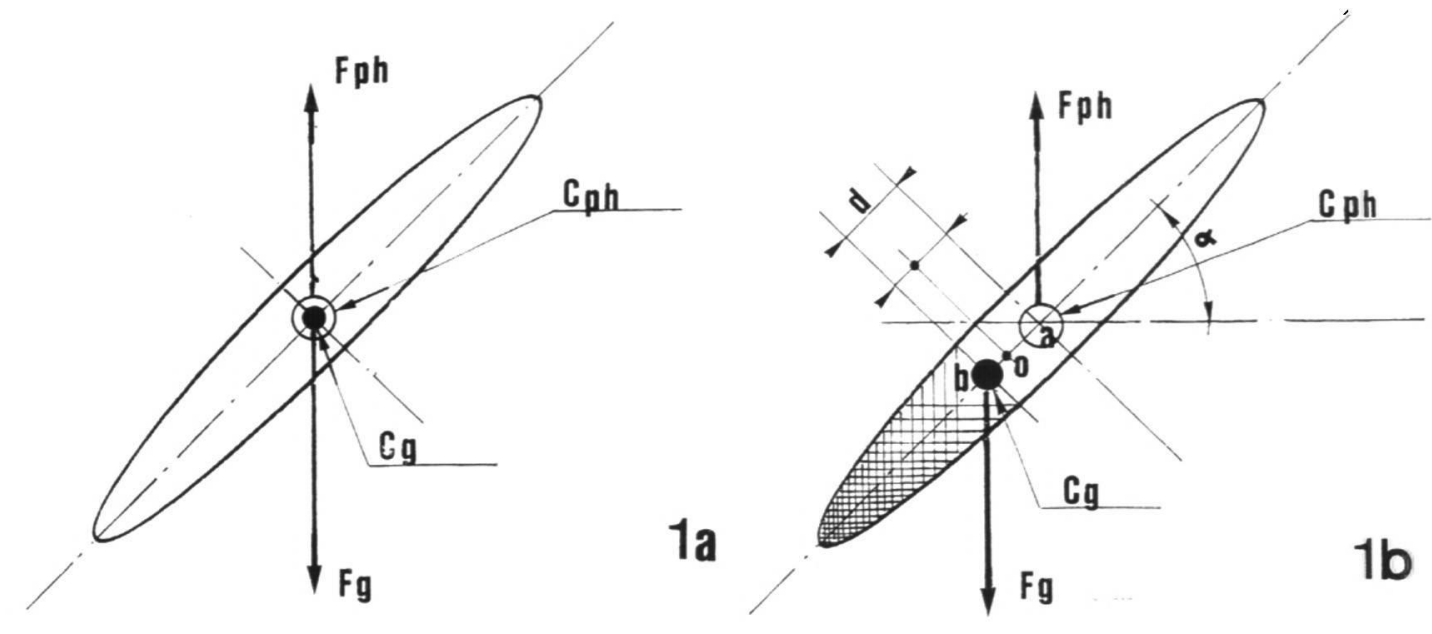

Figure 1 : Schéma de la répartition des forces de gravitation (Fg) et de la pression hydrostatique (Fph) dans un liquide homogène.

1a) Pour un corps homogène, le centre de gravité $(\mathrm{Cg})$ et le centre de poussée (Cph) sont confondus.

1b) Pour un corps hétérogène, le centre de gravité $(\mathrm{Cg})$ et le centre de poussée (Cph) sont séparés par la distance (d).

Figure 1 : Distribution diagram of the forces of gravitation (Fg) and hydrostatic pressure (Fph) in an homogeneous liquid.

1a) For an homogeneous body, the centre of gravity $(\mathrm{Cg})$ and the centre of pressure (Cph) are merged.

1b) For an heterogeneous body, the centre of gravity $(\mathrm{Cg})$ and the centre of pressure (Cph) are separated by the distance (d).

\section{MATÉRIEL ET MÉTHODES}

Pour obtenir des résultats sans ambiguïté et nous assurer de l'indépendance de ces résultats, il nous est apparu nécessaire et indispensable de réaliser ce travail en deux sections indépendantes:

a) Les observations et les mesures de temps de rotation polaire verticale des diatomées. D'une part, les observations ont été conduites de telle manière à n'avoir aucune interférence avec l'observateur, dès l'instant où la diatomée était libérée dans l'espace aquatique ; d'autre part, aucun calcul de force n'a été effectué à partir des mesures de rotation.

b) Le calcul des forces. Ce calcul a été réalisé entièrement sur un modèle théorique, en incluant seulement le résultat brut des observations des mouvements des diatomées vivantes.

Le matériel de laboratoire et les méthodes sont ceux utilisés pour la mesure de la densité des diatomées (BERTRAND, 1993a). Le microscope est un appareil dont l'ensemble platine et objectif peut basculer de $90^{\circ}$ de manière à orienter la platine verticalement, l'oculaire est remplacé par un capteur d'images vidéo CCD en liaison avec un écran de contrôle sur lequel est adaptée une grille de mesure des angles. En outre, le système d'éclairage a été muni d'un filtre anticalorique KODAK 301A absorbant les IR courants, pour stabiliser la température et éviter les courants de convection thermique. Les observations ont été exécutées à température constante de $20^{\circ} \mathrm{C}$, avec un objectif à sec $\times 10^{\circ}$ donnant un grossissement de 350 fois sur l'écran. Un réservoir de mesure a été fabriqué. Le matériel vivant : Navicula radiosa a été choisi pour sa forme très élancée et sa grande symétrie. Les méthodes de mesure des angles de pivotement sont semblables à celles des mesures de densité. Les méthodes de calcul sont issues des cours de mécanique statique (LARALDE, 1981). 


\section{OBSERVATIONS ET RÉSULTATS}

Une étude des pivotements polaires verticaux a été réalisée sur 12 espèces de diatomées raphidées diversifiées (Tableau I).

Tableau I : Temps de rotation sur $90^{\circ}$ des diatomées exécutant un pivotement polaire vertical.

Table I : $90^{\circ}$ rotation elapse time of diatoms turning round in a polar vertical movement.

\section{Pivotements polaires des diatomées}

(Temps de réalisation des pivotements - Mesures prises sur vidéo)

\section{Espèces}

Stauroneis phoenicentron

Navicula radiosa

Navicula radiosa

Navicula radiosa

Pinnularia viridis

Pinnularia major

Nitzschia tryblionella

Nitzschia tryblionella

Nitzschia tryblionella

Nitzschia filiformis

Nitzschia sigma

Nitzschia vitrea

Eunotia pectinalis

Eunotia pectinalis

Gomphoneis minuta

Entomoneis alata

Gyrosigma acuminatum

\section{Réf. vidéo}

V1 - 100

V1 -141

V1 -162

V8 -18

V1 -148

V8 - 46

V1 -182

$\mathrm{V} 1-182$

V6 -72

V4 -27

V4 -68

V6 - 56

V2 - 80

V8 -135

V6 - 88

V $8-43$

V1 - 3
Temps / $90^{\circ}$ Orientation

$$
\begin{gathered}
5,5 \text { m.apic } \\
4 \text { m.apic } \\
2,16 \text { m.apic } \\
\text { 1,16 m.apic } \\
4 \text { m.apic } \\
\text { 4,6 d.apic } \\
9 \text { m.apic } \\
8 \text { d.apic } \\
\text { 3,66 d.apic } \\
\text { 1,3 d.apic } \\
\text { 4,15 m.apic } \\
11 \text { d.apic } \\
\text { 1,25 m.transp. } \\
\text { 1,75 d.transp. } \\
\text { 2,4 m.apic } \\
8 \text { m.apic } \\
\text { 1,8 d.apic }
\end{gathered}
$$

Les 17 temps de rotation de 0 à $90^{\circ}$ en montée (m.apic.) et de descente (d.apic.), relevés sur vidéo, donnent une moyenne de 4,34 secondes. Cependant, on remarque que $72 \%$ des mesures sont situées entre 0 et 4,34 secondes. D'autre part, la moyenne des temps de rotation de descente se situe à 4,58 secondes, avec un écart à la moyenne générale de $+0,24$ secondes, alors que la moyenne des temps de montée est de 4,16 secondes, soit un écart à la moyenne générale de - 0,18 secondes. Les différences très faibles, étant toutes à l'intérieur de l'écart-type, sont non significatives. Toutefois, ceci nous a permis de choisir comme diatomée-test Navicula radiosa, car les temps de rotation (1,16 à 4 secondes) se situent dans le groupe de mesure de 0 à 4,34 secondes pour effectuer $90^{\circ}$ de rotation.

Cependant, il était nécessaire de contrôler l'hétérogénéité de cette diatomée. En effet, s'il est acquis que les divers constituants de la cellule sont de densités différentes (REYNOLDS, 1984) : hydrates de carbone $1,5 \mathrm{gcm}^{-3}$, protéines $1,3 \mathrm{gcm}^{-3}$, acide nucléique $1,7 \mathrm{gcm}^{-3}$, polyphosphates $2,5 \mathrm{gcm}^{-3}$, silice hydratée $2,6 \mathrm{gcm}^{-3}$, lipides $0,86 \mathrm{gcm}^{-3}$ (SARGENT, 1976), cytoplasme $1,05 \mathrm{gcm}^{-3}$, ceux-ci peuvent être disposés symétriquement et avoir un centre de gravité confondu avec le centre de poussée. II était donc indispensable de vérifier la position géométrique de quelques inclusions telles le noyau et les chloroplastes. Pour cette répartition des masses internes, la position des éléments a été vérifiée sur $200 \mathrm{~N}$. radiosa. Les écarts du noyau par rapport au centre de symétrie (Fig. 2a) vont jusqu'à $6 \mu \mathrm{m}$ et seulement $17 \%$ ne montrent pas d'écart. Quant aux chloroplastes (Fig. 2b), l'écart va jusqu'à $9,5 \mu \mathrm{m}$ et, là également, $15 \%$ seulement ne montrent pas d'écart. 


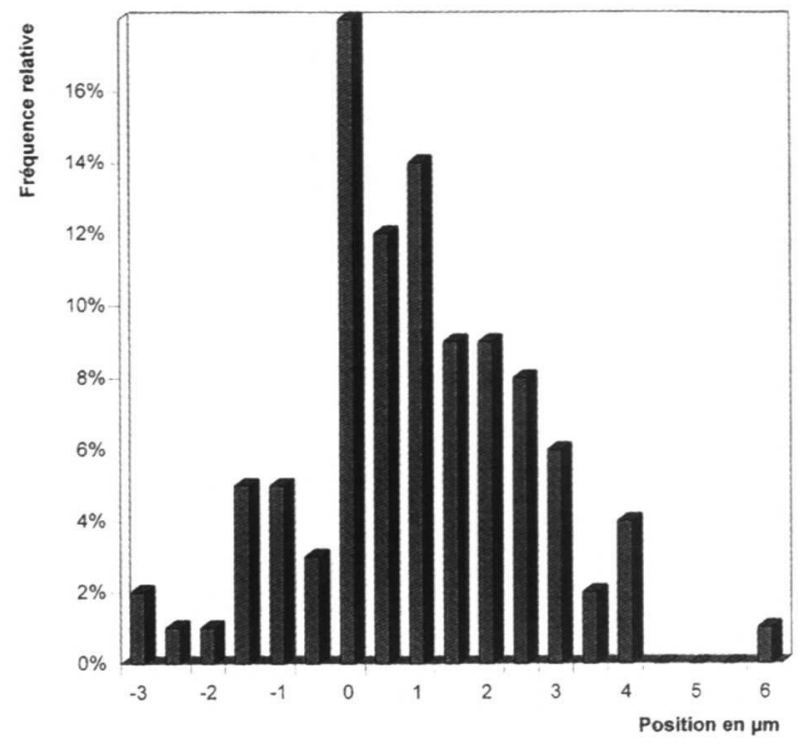

$\underline{2 b}$

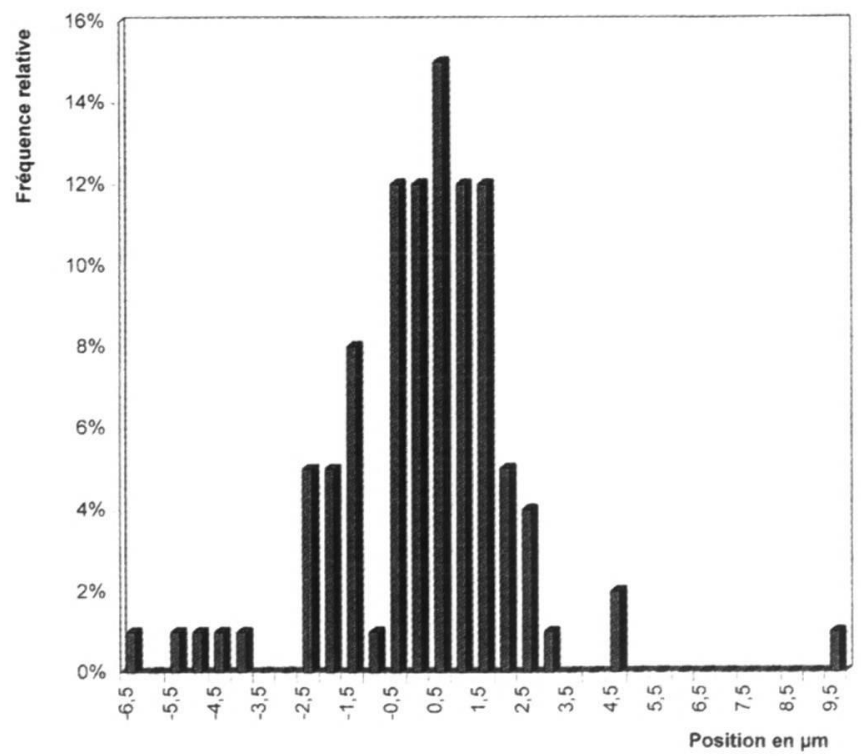

Figure 2 : 2a) Distance du centre de gravité du noyau par rapport au centre de poussée de Navicula radiosa.

2b) Distance du centre de gravité des chloroplastes par rapport au centre de poussée de Navicula radiosa.

Figure 2 :2a) Distance between the centre of gravity of nucleus and the centre of pressure of Navicula radiosa.

2b) Distance between the centre of gravity of chloroplasts and the centre of pressure of Navicula radiosa.

Nous avons bien la confirmation de l'hétérogénéité et de la dissymétrie interne de $N$. radiosa. Elle répond parfaitement à notre exigence, pour vérifier l'influence de la pression hydrostatique. 
Afin d'isoler l'influence seule de la force hydrostatique sur la dissymétrie, nous avons observé les mouvements des diatomées tuées, donc non mobiles par elles-mêmes, dans l'eau, lors de la chute libre. Le test a été réalisé en mesurant les variations angulaires d'orientation spatiale, lors de la chute sur une profondeur variant de 1000 à 1500 microns, de $100 \mathrm{~N}$. radiosa. L'échantillon comprenait des diatomées de longueurs comprises entre 55 et 80 microns, avec une prédominance $(50 \%)$ entre 65 et 70 microns. L'orientation de départ de mesure des angles varie de $0^{\circ}$ (diatomée verticale) à $90^{\circ}$ (diatomée horizontale). L'angle de départ et l'angle d'arrivée sont notés et leur différence, positive ou négative, donne l'angle de pivotement pour une distance de chute de 500 microns, à une vitesse moyenne de $23,28 \mu \mathrm{ms}^{-1}$. La répartition des angles de pivotement est significative (Fig. 3), $32 \%$ des diatomées ne changent pas d'orientation au cours de leur chute (angle de pivotement $=0^{\circ}$ ), quel que soit l'angle de départ (Fig. 4), mais $51 \%$ pivotent de 5 à $15^{\circ}$ et certaines jusqu'à $65^{\circ}(1 \%)$.

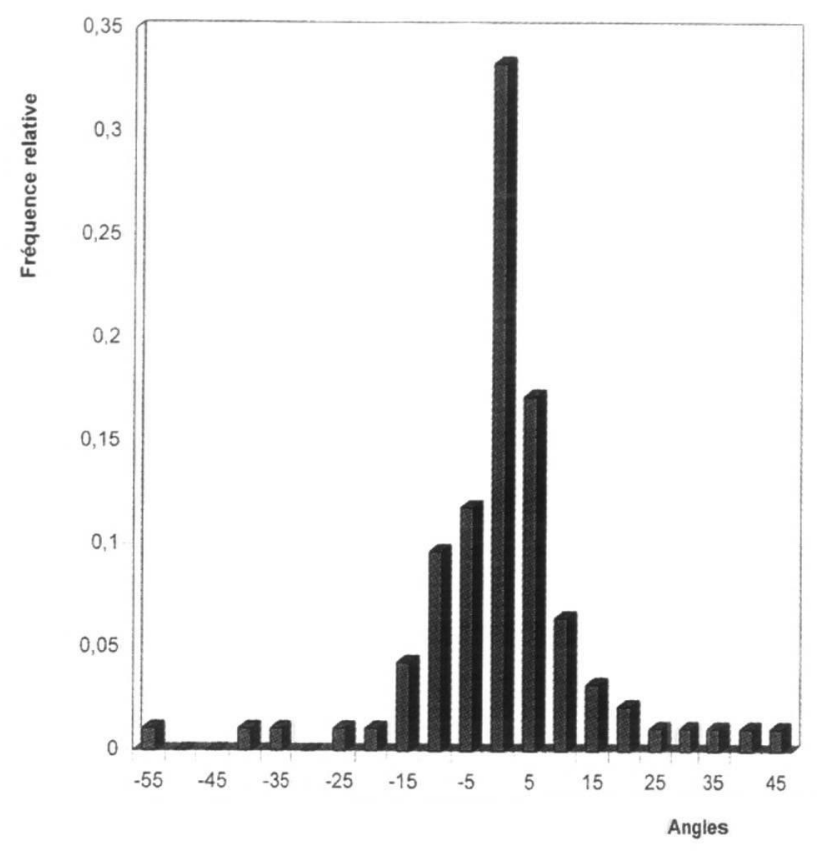

:igure 3 : Répartition en valeur relative des angles de pivotement des diatomées complètes mortes.

تigure 3 : Distribution in relative value of rotation angles of complete dead diatoms.

Figure 4 : Répartition des angles de départ, lors de leur chute, des diatomées complètes mortcs dans la cuve de mesure.

Figure 4 : Distribution of angles at the beginning of the fall of complete dead diatoms in the measurement tank.

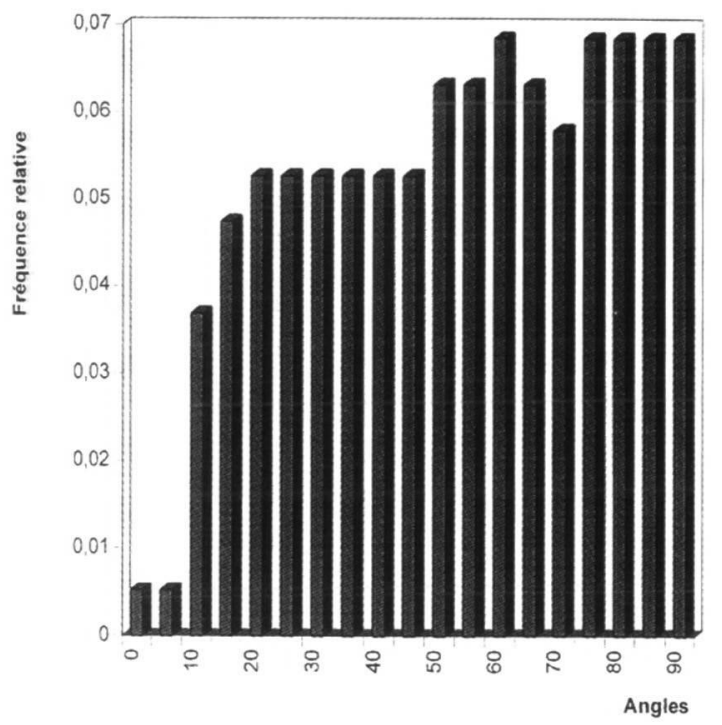


D'autre part, les caractéristiques géométriques et d'orientation spatiale de départ des diatomées n'ont aucune influence sur les angles de pivotement puisque le coefficient de corrélation " longueur de la diatomée / angle de départ " est de 0,053 , celui de "longueur de la diatomée / angle de pivotement " de 0,087 et celui de " angle de départ / angle de pivotement " de 0,282 . Cependant, on peut se demander si la forme hydrodynamique de la diatomée n'a pas une action prédominante dans la rotation. Ceci nous a conduits à effectuer les mesures d'orientation, dans les mêmes conditions que $N$. radiosa, sur 200 Fragilaria ulna, de longueurs comprises entre 90 et $270 \mu \mathrm{m}$. Nous avons obtenu des résultats sensiblement équivalents : $45 \%$ des diatomées dont l'orientation ne varie pas, $34 \%$ dont l'orientation varie de 5 à $15^{\circ}$, et $1 \%$ vont jusqu'à $65^{\circ}$. De plus, aucune tendance n'a été relevée vers une orientation plus verticale. Ceci lève donc le doute quant à l'influence de la forme, tout au moins pour une hauteur de chute de $500 \mu \mathrm{m}$.

Toutefois, il était indispensable de comparer ces résultats avec ceux d'un test dont les diatomées sont parfaitement homogènes et symétriques. Ce test a été réalisé à l'aide de 100 frustules complètes de $N$. radiosa, préparées dans $\mathrm{H}_{2} \mathrm{O}_{2}$. La figure 5 montre sans ambiguïté, pour les frustules, le regroupement autour de la valeur $0^{\circ}$ des angles de pivotement, avec une prédominance remarquable de l'absence de rotation pour $62 \%$ des frustules. Nous avons ainsi la confirmation de l'influence certaine de la pression hydrostatique, lors de la chute des diatomées entières mortes du premier test.

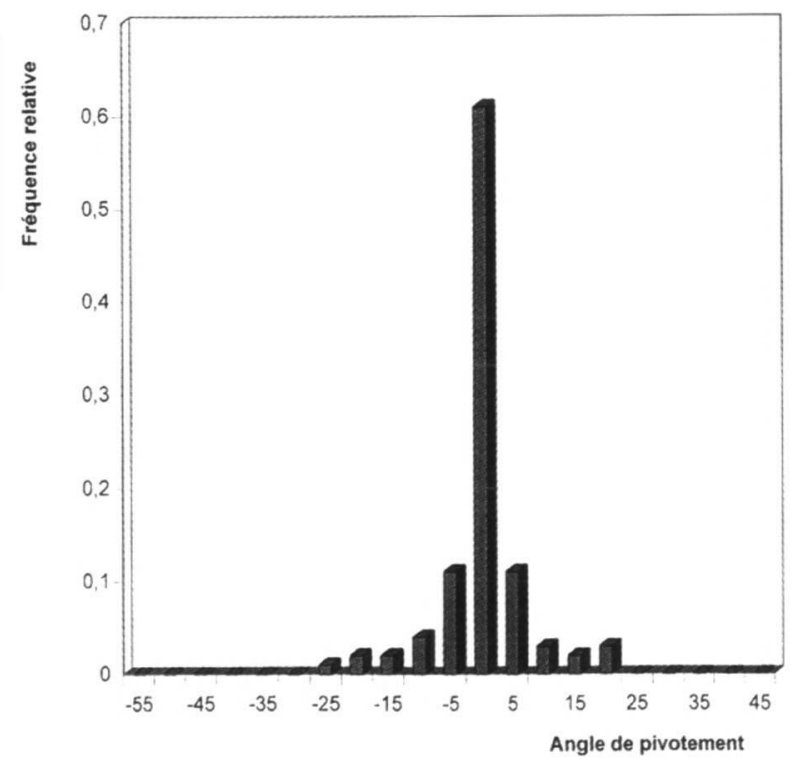

Figure 5 : Répartition en valeur relative des angles de pivotement des frustules.

Figure 5 : Distribution in relative value of rotation angles of frustules.

La comparaison des vitesses de rotation des diatomées entières mortes et des diatomées vivantes donne une bonne indication pour juger exactement de l'influence réelle de la force hydrostatique. En prenant comme base de calcul, pour les diatomées entières mortes, $15^{\circ}$ de rotation pour $500 \mu \mathrm{m}$ de chute (angle de rotation maximum pour $66,6 \%$ de la population étudiée), à la vitesse de $23,28 \mu \mathrm{ms}^{-1}$, nous obtenons 0,0019 tour par seconde.

Ces résultats sont à comparer au maximum des temps de rotation “ volontaire " de N. radiosa vivante, de 4 secondes pour $90^{\circ}$, soit 0,0625 ts $^{-1}$.

Rapport vitesse des diatomées mortes / vitesse des diatomées vivantes $=0,031$.

La force hydrostatique n'entrerait donc que pour environ $3 \%$ dans les vitesses obtenues par la diatomée pour effectuer le pivotement polaire vertical. 


\section{ANALYSE}

L'observation des comportements à travers les vitesses de rotation des diatomées vivantes et mortes nous a permis de cerner une partie des phénomènes. Une autre partie concerne les efforts développés sous la force hydrostatique, pour la rotation du matériel mort et des diatomées vivantes sous leur propre "volonté ". Préalablement, un certain nombre de calculs s'imposent : a) mesure de la densité, b) calcul du centre de gravité d'une diatomée, c) calcul des efforts. Nous avons ensuite calculé le rapport "efforts fournis par la diatomée vivante dissymétrique / efforts fournis par une diatomée symétrique ", indépendamment des vitesses observées, ce qui nous renseigne sur l'importance de la force hydrostatique. La comparaison avec le résultat de l'analyse de vitesse nous renseigne alors sur la validité des calculs effectués.

\section{a) Mesure de la densité}

Cette mesure effectuée suivant la méthode des vitesses différentielles (BERTRAND, 1992) permet, par la résolution de l'équation de la droite de régression, d'obtenir la valeur de la densité $1,44 \mathrm{~g} / \mathrm{cm}^{-3}$ et la vitesse de chute dans l'eau à $23,28 \mu \mathrm{ms}^{-1}$.

\section{b) Calcul du centre de gravité de $N$. radiosa}

II a été créé un modèle (Fig. 6), en tenant compte des observations précédentes concernant la répartition interne et externe des masses des éléments cellulaires, ainsi que leur densité estimée. Nous avons inclus, outre le noyau et les chloroplastes, 2 gouttes lipidiques fortement décentrées et dissymétriques. Nous avons bien conscience de l'imperfection de ce modèle, car les données concernant la densité des éléments constitutifs se rapportent souvent à des molécules et non à des ensembles complexes, comme par exemple les acides nucléiques par rapport au noyau. Par ailleurs, en ce qui concerne les chloroplastes, leur densité a été estimée, car nous n'avons trouvé aucune valeur dans la littérature scientifique. En effet, plusieurs auteurs donnent des gradients de centrifugation, mais aucune valeur de la densité : JACKSON et al. (1978), CHUA et SCHMIDT (1978), TAHABE et al. (1979).

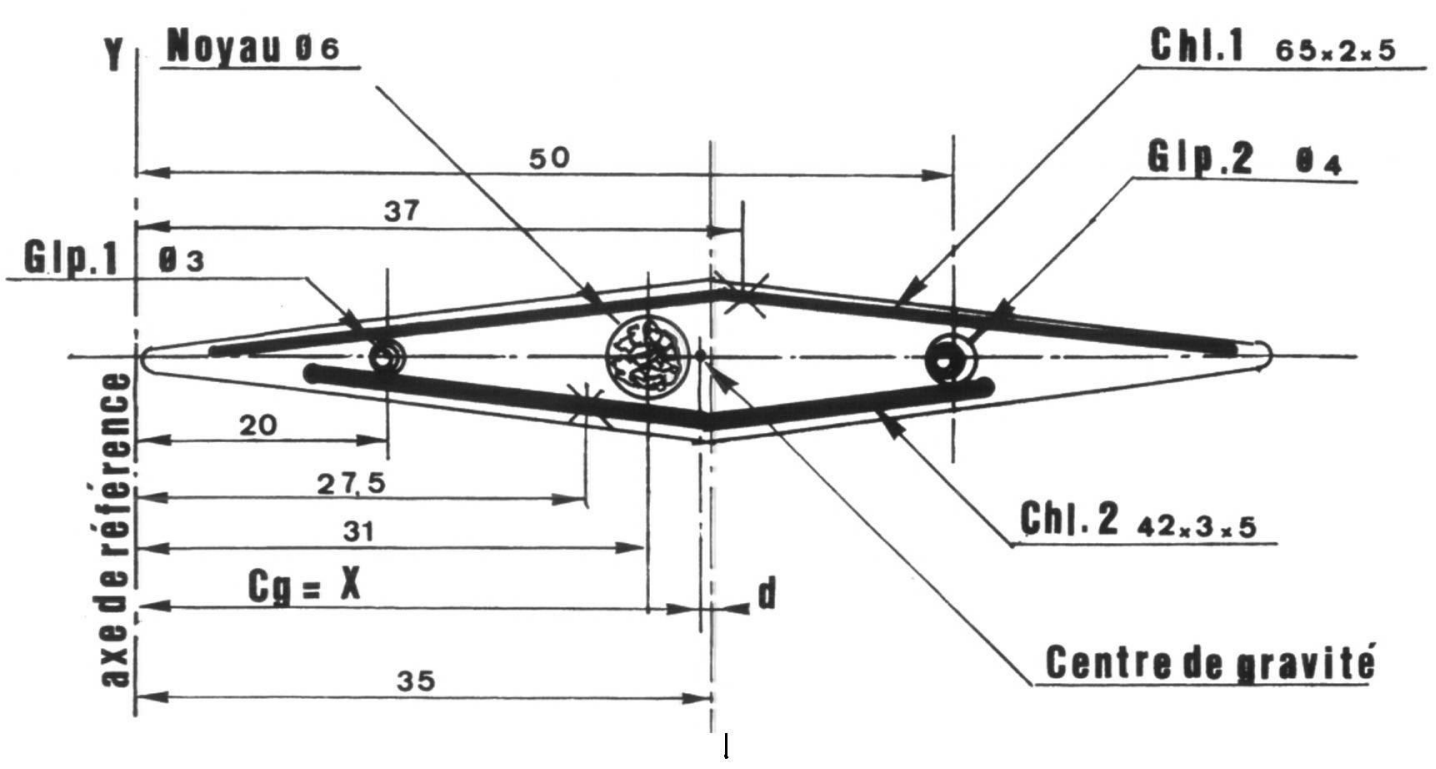

Figure 6 : Modèle de Navicula radiosa (dimensions $70 \times 11 \times 6 \mu \mathrm{m}$ ). Répartition des éléments constitutifs, on note : Chl 1 et Chl 2 = chloroplastes, Glp 1 et Glp 2 = gouttes lipidiques, $\mathrm{Cg}=\mathrm{X}$ : distance du centre de gravité à l'axe $\mathrm{Y}$. Les dimensions sont en $\mu \mathrm{m}$.

Figure 6 : Navicula radiosa model (size $70 \times 11 \times 6 \mu \mathrm{m}$ ). Distribution of constituting elements : Chl 1 and $\mathrm{Chl} 2$ = chloroplasts, Glp 1 and Glp 2 = lipiditic drops, $\mathrm{Cg}=\mathrm{X}:$ distance between the centre of gravity and the $Y$ axis. The dimensions are measured in $\mu \mathrm{m}$. 
Le calcul est effectué à l'aide du théorème des Moments (LARALDE, 1981). Nous avons considéré que seuls les Moments par rapport à l'axe des $Y$ étaient nécessaires et suffisants pour l'analyse. La position du centre de gravité a été calculée ainsi que l'écart (d) du centre de gravité $\mathrm{Cg}$ au centre de poussée, soit $0,523 \mu \mathrm{m}$.

\section{c) Calcul des forces}

Celui-ci s'effectue également à l'aide du théorème des Moments, en considérant 4 équations de base dont la somme est égale à 0 : équations par rapport aux 3 axes $x, y, z$, plus une équation par rapport à un point. Les réactions aux appuis n'étant pas nécessaires, nous garderons seulement cette dernière qui se résume ainsi :

Somme des Moments par rapport à un point $=0$, d'où (Fig. 7) :

$F_{1}=$ force de traction exercée sur un bras de levier de $3 \mu \mathrm{m}$ par la diatomée vivante et ayant un centre de gravité distant de (d) du centre de poussée ;

$F_{2}=$ force de traction exercée sur un bras de levier de $3 \mu \mathrm{m}$ par une diatomée vivante, dont le centre de gravité et le centre de poussée sont confondus, (non représenté).

\section{Diatomée horizontale - vue connective}

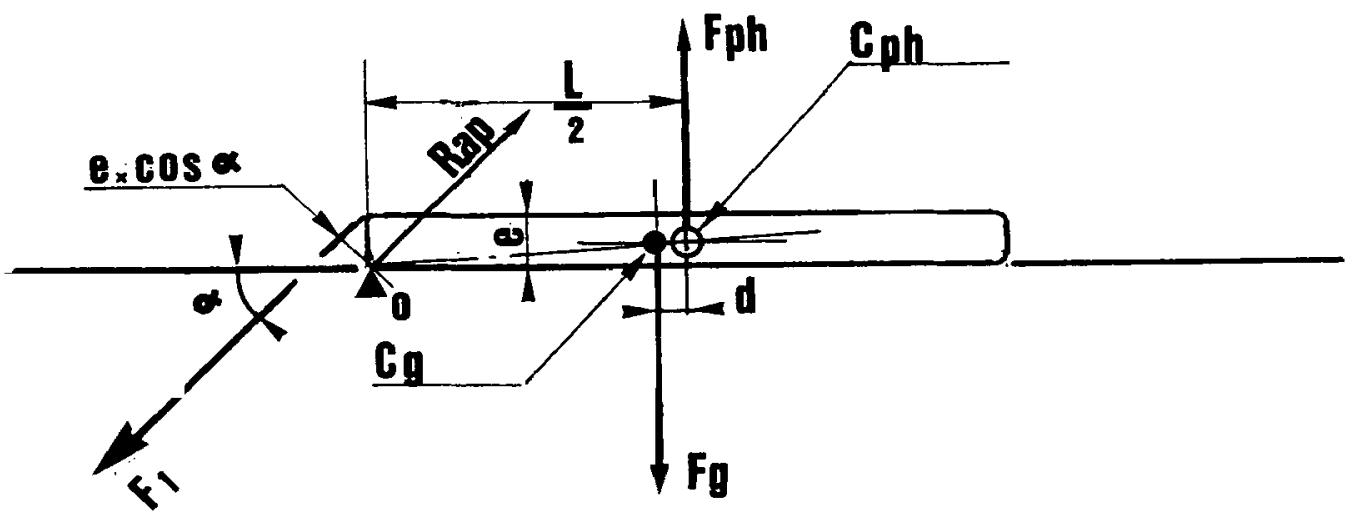

Figure 7 : Schéma de répartition des forces en équilibre par rapport à un point, pour le début du pivotement polaire vertical de Navicula radiosa.

Figure 7 : Distribution diagram of the forces balancing, for the beginning of the polar vertical rotation of Navicula radiosa.

Les forces $F_{1}$ et $F_{2}$ sont admises selon une hypothèse basée sur les observations des pivotements polaires verticaux de Eunotia pectinalis (BERTRAND, 1993b).

La résolution de ces équations simples donne un rapport de F1 - F2 / F2 = à 4,9\%, pour un écart d égal à $0,523 . \mu \mathrm{m}$, calculé précédemment.

La force hydrostatique n'entrerait que pour $5 \%$ dans les efforts fournis par la diatomée pour effectuer le pivotement polaire vertical.

\section{DISCUSSION - CONCLUSION}

La concordance entre les valeurs des vitesses mesurées et les forces calculées doit être prise avec beaucoup de prudence car, d'une part, ces résultats décrivent deux phénomènes distincts qui ne sont pas liés par une équation et, d'autre part, on peut toujours objecter que le modèle peut évoluer suivant les besoins de la démonstration. Ce qui n'est pas le cas ici, puisque 
nous avons pris la précaution de prendre les dimensions moyennes des diatomées mesurées. Nous devons également nous interroger sur la validité et les limites de ces résultats. Autrement dit, sont-ils plausibles?

Nous avons recalculé, à l'aide du modèle, une diatomée fictive anormale et improbable, dont les éléments constitutifs sont très excentrés : le noyau est décalé du maximum observé, soit $6 \mu \mathrm{m}$; les 2 chloroplastes sont regroupés dans une seule moitié de la diatomée ; nous avons supprimé la grosse goutte lipidique et conservé la petite. Les résultats sont intéressants : la densité a peu évolué, passant de 1,44 à $1,457 \mathrm{gcm}^{-3}$, tandis que l'écart du centre de gravité au centre de poussée passe de 0,523 à $1,205 \mu \mathrm{m}$. Le calcul des efforts montre alors que la pression hydrostatique représente $11 \%$ de la force nécessaire pour une diatomée à centre de gravité coaxial avec le centre de poussée. Si maintenant nous retraduisons ces $11 \%$ dans le cadre du test sur les angles de pivotement, nous trouvons une vitesse de rotation de 0,0068 ts $^{-1}$ correspondant à $55^{\circ}$ pour la chute de $500 \mu \mathrm{m}$, soit le maximum observé pour une seule diatomée du test. La concordance semble plausible.

En conclusion, nous pouvons admettre sans trop d'erreur que la pression hydrostatique a une action insignifiante dans le cas de pivotement polaire vertical des diatomées (elle ne dépasse pas $5 \%$ des efforts développés pour exécuter le mouvement et atteint exceptionnellement $10 \%$, pour des cellules anormales). Par ailleurs, l'influence de la pression hydrostatique sur les mouvements transapicaux des diatomées est certainement nulle, puisque la distance du point d'appui au centre de gravité est 7 à 8 fois plus petite. D'autre part, il semble que nous pouvons admettre la validité des calculs de biomécanique, car ceux-ci paraissent confirmer les observations, à la condition que les lois de la mécanique générale soient bien respectées et appliquées, tout au moins dans les limites des dimensions des diatomées. Bien entendu, les résultats de ces calculs sont des ordres de grandeur, mais ils peuvent malgré cela permettre de se faire une idée correcte des relations diatomée - substrat.

\section{REMERCIEMENTS}

L'auteur remercie particulièrement les lecteurs, pour leurs suggestions judicieuses, et plus spécialement son épouse, pour le travail ingrat de secrétariat et de correction du manuscrit.

\section{BIBLIOGRAPHIE}

BERTRAND J., 1991. Mouvement des diatomées. I - L'équilibre dynamique chez Rhoicosphenia abbreviata. Cryptogamie, Algol., 12 (1), 11-29.

BERTRAND J., 1992. Mouvement des diatomées. II - Synthèse des mouvements. Cryptogamie, Algol., 13 (1), 49-71.

BERTRAND J., 1993a. La densité des diatomées benthiques : une nouvelle méthode de mesure (vitesse différentielle). Cryptogamie, Algol., 14 (1), 21-35.

BERTRAND J., 1993b. Mouvement des diatomées. III - Le pivotement polaire vertical de Eunotia pectinalis (Kütz.) Rab. Essais de quantification des forces. Cryptogamie, Algol., 14 (4), 157-172.

BERTRAND J., 1995. Mouvement des diatomées. IV - Le mouvement transapical de Cocconeis pediculus, Ehrenberg. Cryptogamie, Algol., 16 (1), 1-20.

CHUA N.H., SCHMIDT G.W., 1978. Post-translational transport into intact chloroplasts of a precursor to the small subunit of ribulose - 1,5- bisphosphate carboxylase. Proc. Nat. Acad. Sci., USA, 75, 6110-6114.

EDGAR L.A., 1982. Diatom locomotion : a consideration of movement in a highly viscous situation. Brit. Phycol. J., 17, 243-251.

FINER T.J., SIMMONS R.M., SPUDICH J.A., 1994. Single myosin molecule mechanics : piconewton and nanometre steps. Nature, 368, 113-118. 
HARPER M.A., HARPER J.F., 1967. Measurements of diatom adhesion and their relationships with movement. Brit. Phycol. Bull., 3 (2), 195-207.

ISHIJIMA A., DOI T., SAKURADA K., YANAGIDA T., 1991. Sub-piconewton force fluctuation of actomyosine in vitro. Nature, 352, 305-306.

JACKSON C., DENCH J.E., HALLIWELL B., 1978. Separation of chloroplasts from mitochondria utilising silica sol gradient centrifugation. The Wolfson Conference, University of Surrey.

KUO S.C., SHEETZM.P., 1993. Force in single kinesin molecules measured with optical tweezers. Science, 260, 232-234.

LARALDE J.P., 1981. Mécanique statique. Méthode graphique. Masson, Paris, 200 p.

LENGELER J.W., 1990. La nage des bactéries. La recherche, 21, 20-28.

REYNOLDS C.S., 1984. The ecology of freshwater phytoplankton. Cambridge University Press, $374 \mathrm{p}$.

SARGENT J.R., 1976. The structure, metabolism and function of lipids in marine organisms. In MALINS D.C., SARGENT J.R. (eds.), Biochemical and Biophysical Perspectives in Marine Biology, 150-212.

SMAYDA T.J., 1970. The suspension and sinking of phytoplankton in the sea. Oceanogr. Mar. Biol. Ann. Rev., 8, 353-414.

SMAYDA T.J., BOLEYN B.J., 1965. Experimental observations and flotation of marine diatoms. 1 - Thalassiosira cf. nana, Thalassiosira rotula and Nitzschia seriata. Limnol. Océanogr., 10, 499-509.

TAHABE T., NISHIMURU M., AKAAZAWA T., 1979. Isolation of intact chloroplasts from spinach by leaf centrifugation in gradients of modified silica " Percoll ". Agric. Biol. Chem., 43, 2137-2142.

WALSBY A.E., REYNOLDS C.S., 1980. Sinking and floating. In MORRIS I. (ed.), Physiological Ecology of phytoplankton, Blackwell Publ., 371-412. 